\title{
The new Ghent criteria for Marfan syndrome: what do they change?
}

Faivre L, Collod-Beroud G, Adès L, Arbustini E, Child A, Callewaert BL, Loeys B, Binquet C, Gautier E, Mayer K, Arslan-Kirchner M, Grasso M, Beroud C, Hamroun D, Bonithon-Kopp C, Plauchu H, Robinson PN, De Backer J, Coucke P, Francke U, Bouchot O, Wolf JE, Stheneur C, Hanna N, Detaint D, De Paepe A, Boileau C, Jondeau G.

The diagnosis of Marfan syndrome (MFS) is challenging and international criteria have been proposed. The 1996 Ghent criteria were adopted worldwide, but new diagnostic criteria for MFS were released in 2010, giving more weight to aortic root aneurysm and ectopia lentis. We aimed to compare the diagnosis reached by applying this new nosology $v s$ the Ghent nosology in a well-known series of 1009 probands defined by the presence of an FBN1 mutation. A total of 842 patients could be classified as MFS according to the new nosology (83\%) as compared to $894(89 \%)$ according to the 1996 Ghent criteria. The remaining $17 \%$ would be classified as ectopia lentis syndrome (ELS), mitral valve prolapse syndrome or mitral valve, aorta, skeleton and skin (MASS) syndrome, or potential MFS in patients aged less than 20 years. Taking into account the median age at last follow-up (29 years), the possibility has to be considered that these patients would go on to develop classic MFS with time. Although the number of patients for a given diagnosis differed only slightly, the new nosology led to a different diagnosis in $15 \%$ of cases. Indeed, $10 \%$ of MFS patients were reclassified as ELS or MASS in the absence of aortic dilatation; conversely, 5\% were reclassified as MFS in the presence of aortic dilatation. The nosology is easier to apply because the systemic score is helpful to reach the diagnosis of MFS only in a minority of patients. Diagnostic criteria should be a flexible and dynamic tool so that reclassification of patients with alternative diagnosis is possible, requiring regular clinical and aortic follow-up.

\section{Conflict of interest}

The authors declare no conflict of interest.

\author{
L Faivre ${ }^{\mathrm{a}, \mathrm{b}, \mathrm{c}}$, \\ G Collod-Beroud $^{\mathrm{d}, \mathrm{e}}$, L Adès $\mathrm{s}^{\mathrm{f}, \mathrm{g}, \mathrm{h}}$, \\ E Arbustini', A Childi, \\ BL Callewaert ${ }^{k}$, B Loeys ${ }^{k, l}$, \\ C Binquet ${ }^{b, c, m}, E$ Gautier ${ }^{b, c, m}$, \\ K Mayer ${ }^{\mathrm{n}}$, M Arslan-Kirchner ${ }^{\circ}$, \\ M Grassoi, C Beroud ${ }^{d, e, p}$, \\ D Hamroun ${ }^{p}$, C Bonithon- $^{-}$ \\ Kopp ${ }^{b, c, m}$, H Plauchuq, PN \\ Robinson ${ }^{r}$, J De Backerk, $P$ \\ Coucke $^{k}$, U Franckes, $O$ \\ Bouchot $^{t}$, JE Wolf ${ }^{\mathrm{u}}$, C \\ Stheneurv, N Hannaw, D \\ Detaint" , A De Paepe ${ }^{k}$, C \\ Boileau $^{\mathrm{v}, \mathrm{w}}$ and $\mathrm{G}$ Jondeau ${ }^{\mathrm{v}}$
}

${ }^{a}$ Centre de Génétique et Centre de Référence Anomalies du Développement et Syndromes Malformatifs, CHU Dijon, Dijon, France, ${ }^{b} \mathrm{CHRU}$ Dijon, Centre d'investigation clinique - épidémiologie clinique/essais cliniques, Dijon, France, 'Equipe GAD, Univ de Bourgogne, Dijon, France, dINSERM, U827, Montpellier, France, eUniv Montpellier I, Montpellier, France, ${ }^{\mathrm{f}}$ Marfan Research Group, The Children's Hospital at Westmead, Sydney, NSW, Australia, 9Discipline of Paediatrics and Child Health, University of Sydney, Sydney, NSW, Australia,

hDepartment of Clinical Genetics, The Children's Hospital at Westmead, Sydney, NSW, Australia, 'Centre for Heritable Cardiovascular Diseases, IRCCS Foundation Policlinico San Matteo, Pavia, Italy, 'Department of Cardiological Sciences, St. George's Hospital, London, UK, k'Center for Medical Genetics, Ghent University Hospital, Belgium, 'Center for Medical Genetics, Antwerp University Hospital, Antwerp-Edegem, Belgium, mInserm, CIE1, Dijon, France, ${ }^{n}$ Center for Human Genetics and Laboratory Medicine, Martinsried, Germany, Institut für Humangenetik, Hannover, Germany, PLaboratoire de Biologie Moléculaire, CHU Montpellier, Montpellier, France, aService de Génétique, Hôtel Dieu, Lyon, France, 'Institut für Medizinische Genetik, Universitätsmedizin Charité, Berlin, 
Germany, sDepartments of Genetics and

Pediatrics, Stanford University Medical Center, Stanford, CA, USA, ${ }^{\mathrm{t} C h i r u r g i e}$ cardiovasculaire and "Cardiologie, $\mathrm{CHU}$ Dijon, Dijon, France, ${ }^{\vee}$ Centre de Référence Syndrome de Marfan et apparentés, Bichat, AP-HP, Paris,

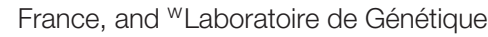
moléculaire, Hôpital Ambroise Paré, Boulogne, France

Key words: Ghent criteria - Marfan syndrome - new criteria - nosology

Corresponding author: Laurence Faivre, MD-PhD, Centre de Génétique, Hôpital d'Enfants, 10, bd Maréchal De Lattre de Tassigny, 21034 Dijon, France. Tel.: +33 3802933 00; fax: +33 3802932 66; e-mail: laurence.faivre@chu-dijon.fr
Marfan syndrome (MFS; MIM\#154700) is a connective tissue disorder, with autosomal dominant inheritance and a prevalence of 1/5000 individuals $(1,2)$, caused by FBN1 mutations. The cardinal features of MFS involve the ocular, cardiovascular and skeletal systems (3). The skin, lung and dura may also be involved. To facilitate accurate communication about the condition among healthcare providers, researchers and patients, as well as to facilitate appropriate patient management and counseling, clinical criteria for the diagnosis of MFS were established $(4,5)$. The initial criteria were proved to be too loose once molecular data became available $(6,7)$. For MFS to be diagnosed in an individual, the revised diagnostic criteria required at least two major systems to be affected (lens dislocation, ascending aortic dilatation or dissection, dural ectasia, a specified combination of skeletal features, a family history or FBN1 mutation) and involvement of at least one other organ system (5). Reviews of patients referred for FBN1 analysis indicated that an FBN1 mutation was identified in $72.5-91 \%$ of patients who fulfilled clinical Ghent criteria $(8-10)$.

Nevertheless, concerns about sensitivity have been raised. In particular, the 1996 Ghent criteria did not take sufficient account of the agedependent nature of some clinical manifestations, making the diagnosis in children sometimes difficult (11), or of the diversity of clinical presentations secondary to FBN1 mutations (12). Also, the 1996 Ghent criteria comprised physical manifestations with poorly validated diagnostic thresholds (e.g. flat feet and pulmonary artery dilatation), (13) or signs that are not systematically screened for (e.g. dural ectasia). The main reason for introducing a new nosology was that the presence of aortic dilatation was not mandatory in a personal or family history to raise the diagnosis of MFS. Indeed, physicians associate the diagnosis of 'MFS' with cardiovascular risk. It has been judged detrimental to diagnose MFS in patients without tangible evidence of cardiovascular risk, because it may restrict patients' access to insurance benefits or career opportunities. Other concerns were the additional financial burden associated with frequent medical care, anxiety or situational depression, unfounded marital or reproductive decisions, the loss of health benefits or the psychosocial stigmatization associated with exercise restriction, which is a particularly important issue during childhood.

As a result, five major changes in the diagnostic guidelines were proposed (Table 1) (14): (i) more weight is given to two cardinal features of MFS, aortic root aneurysm/dissection and ectopia lentis. All the other cardiovascular and ocular manifestations of MFS and findings in other organ systems, such as the skeleton, dura, skin and lungs, contribute to a 'systemic score' (Table 1) that guides diagnosis depending on the presence or absence of aortic disease or ectopia lentis; (ii) a more prominent role is assigned to molecular genetic screening for $F B N 1$ and other relevant genes (e.g. $T G F B R 1$ and 2); (15) (iii) some of the less specific manifestations of MFS were either removed or made less influential in the diagnostic evaluation of patients (e.g. dural ectasia, joint laxity, flat cornea, 
Table 1. Revised Ghent criteria for the diagnosis of Marfan syndrome (MFS) and related conditions (14)

In the absence of a family history:

(1) Ao ( $Z \geq 2)$ AND EL = MFS

(2) Ao $(Z \geq 2)$ AND FBN1 = MFS

(3) Ao $(Z \geq 2)$ AND Syst $(\geq 7$ points $)=$ MFS $^{a}$

(4) EL AND FBN1 with known Ao = MFS

EL with or without Syst AND with an FBN1 not known with Ao or no FBN1 = ELS Ao $(Z<2)$ AND Syst $(\geq 5)$ with at least one skeletal feature without EL $=$ MASS MVP AND Ao $(Z<2)$ AND Syst $(>5)$ without EL $=$ MVPS

In the presence of a family history:

(5) EL AND FH of MFS (as defined above) = MFS

(6) Syst ( $\geq 7$ points) AND FH of MFS (as defined above) $=$ MFS $^{a}$

(7) Ao ( $Z \geq 2$ above 20 years old, $\geq 3$ below 20 years) + FH of MFS (as defined above) $=$ MFS $^{a}$

Systemic score

- Wrist AND thumb sign - $\mathbf{3}$ (Wrist OR thumb sign - 1)

- Pectus carinatum deformity - $\mathbf{2}$ (pectus excavatum or chest asymmetry -1)

- Hindfoot deformity - 2 (plain pes planus - 1)

- Pneumothorax - 2

- Dural ectasia - 2

- Protrusio acetabuli - 2

- Reduced US/LS AND increased arm/height AND no severe scoliosis - 1

- Scoliosis or thoracolumbar kyphosis - 1

- Reduced elbow extension - 1

- Facial features (3/5) - 1 (dolichocephaly, enophtalmos, downslanting palpebral fissures, malar hyoplasia, retrognathia)

- Skin striae - 1

- Myopia >3 diopters - 1

- Mitral valve prolapse (all types) - 1

Maximum total: 20 points; score $\geq 7$ indicates systemic involvement

Ao, aortic diameter at the sinuses of valsalva above indicated Z-score or aortic root dissection; EL, ectopia lentis; ELS, ectopia lentis syndrome; FBN1, fibrillin-1 mutation; FBN1 with known Ao, FBN1 mutation that has been identified in an individual with aortic aneurysm; FH, family history; MASS, myopia, mitral valve prolapse, aortic root dilation, skeletal findings, striae syndrome; MVPS, mitral valve prolapse syndrome; Syst, systemic score; US/LS, upper segment/lower segment ratio; Z, Z-score.

${ }^{a}$ Caveat: without discriminating features of Shprintzen-Goldberg syndrome, Loeys-Dietz syndrome or vascular EDS syndrome.

- if present, then TGFBR1/2 testing, collagen biochemistry, COL3A1 testing

- other conditions/genes will emerge with time.

iris or ciliary muscle hypoplasia, dilatation of the pulmonary artery, mitral annulus calcification and recurrent or inscisional herniae); (iv) the new criteria formalize the concept that additional diagnostic considerations and testing are required if a patient has sufficient findings to satisfy the criteria for MFS but also shows unexpected findings, particularly if they segregate with disease in the family or if they are suggestive of a specific alternative diagnosis (e.g. Shprintzen-Goldberg syndrome, Loeys-Dietz syndrome $(16,17)$ and the vascular form of Ehlers-Danlos syndrome); and (v) the new introduced categories [ectopia lentis syndrome (ELS), myopia, mitral valve prolapse, borderline and non-progressive aortic root dilatation, skeletal findings and striae (MASS) syndrome, mitral valve prolapse syndrome (MVPS)] cannot be used in patients aged less than 20 years, and the term 'potential MFS' is proposed in such patients, to take into account that the phenotype may evolve with time.

Since the 1996 Ghent criteria were adopted worldwide, these new criteria will lead to a new approach in the physicians' evaluation. Moreover, they may modify the diagnosis for a given patient. We chose here to assess the changes engendered by this new classification in a well-characterized series of 1009 probands with a known FBN1 mutation $(12-18)$.

\section{Patients and methods}

A total of 1191 probands diagnosed with type I fibrillinopathy between 1995 and 2005 were identified via the Universal Marfan database - FBN1 (UMD-FBN1; http:/www.umd.be) (19) and the network of participating centers. Inclusion criteria were (1) heterozygosity for a pathogenic $F B N 1$ gene mutation in a patient with a phenotype 
compatible with type I fibrillinopathy and (2) the availability of clinical information. The determination of the pathogenic nature of the mutation has been discussed previously $(18,20)$. Within this cohort, 178 probands were excluded from the study (no clinical data available in 129, insufficient data on cardiovascular, ocular or skeletal involvement in 44 and compound heterozygosity for FBN1 mutations in 5). The patients originated from 38 different countries located across five continents and the majority of inclusions were from referral centers for MFS. The majority (72\%) were white Europeans or of European ancestry, 14\% were from North and South America, 8\% were from Oceania, 4\% were from Asia, and 2\% were from Africa. The clinical data were collected either from a questionnaire sent to referring physicians and clinical investigators or from previous publications wherein sufficient information was available. All questionnaires were collected and referenced by one individual (L. F.) to avoid duplication of patients in the study. The clinical information required included a range of qualitative and quantitative clinical parameters, including cardiovascular, ophthalmological, skeletal, skin, lung and dural manifestations. These patients were collected for the purpose of a large-scale genotype-phenotype correlation study (18), and the cohort was further investigated for other analyses $(11,12,21-23)$.

Each patient was reclassified according to the 2010 Ghent criteria, taking into account their manifestation at last follow-up. Four patients with autosomal dominant Weill-Marchesani syndrome were not assessed because they represented another well-defined type I fibrillinopathy (24-26). A cohort of 1009 patients was thus constituted. The results were compared to those obtained according to the 1996 Ghent criteria. In the overall cohort and for each diagnosis (MFS, ELS, MASS and MVPS), the median age at diagnosis was analyzed, as was the systemic score distribution. The results were analyzed distinguishing between probands aged less than 20 years (named 'children cohort') and those above (named 'adult cohort'). The differences between both classifications were analyzed. By applying the new nosology, authors noticed that patients with a systemic score of less than 7, without aortic dilatation or ectopia lentis, but with a family history of MFS and/or FBN1 mutation already associated with an aortic dilatation, fell into a gap in the new nosology. These patients have been classified as MFS in this study so that patients from the same family and carrying the same FBN1 mutation will reach the same diagnosis.
As this study was retrospective, some items were defined differently in the old and new criteria. For example, in the presence of a family history, a $Z$-score $>3$ for the aortic root is required when an individual is aged less than 20 years. The only information available for all patients in this retrospective study was the presence or absence of aortic root dilatation. When a mutation was not associated with aortic dilatation, we searched the UMD-FBN1 database $(n=19)$ for other patients with the same mutation who did have an aortic phenotype. The differential items were particularly notable for the determination of the systemic score. The new criteria score 1 point for a wrist OR thumb sign and 3 points for a wrist AND thumb sign. As the medical records did not differentiate between these, 3 points were assigned when a patient had arachnodactyly. Similarly, as it was not possible to distinguish between hindfoot deformity and pes planus, 1 point was attributed to all patients with pes planus. The number of facial features was not known and 1 point was given to a patient with compatible facial dysmorphism. One point was attributed to patients with severe myopia, even if the dioptres were not known. Only 292/1009 patients (29\%) were investigated for the presence or absence of dural ectasia. To evaluate the impact of this methodology, as well as the easier applicability of the new Ghent criteria, a flow chart was made to evaluate the number of patients in which the determination of the systemic score was mandatory to raise the diagnosis of MFS. The distribution of types of mutations according to the different types of presentation (MFS, ELS, MASS, MVPS and potential MFS) was determined.

\section{Results}

The median age at diagnosis of the probands was 20 years [interquartile range (IQR) 9-33]. The ages at last follow-up ranged from birth to 72 years, with a median age of 29 years [IQR 15-40], and 354 patients were aged less than 20 years $(35 \%)$. Fifty-four percent of patients were male. The characteristics of the cohort according to the 1996 Ghent criteria have previously been reported (12).

The distribution of diagnoses according to the old and new Ghent criteria is shown in Fig. 1a-c in patients aged 20 years or above and in Fig. $2 \mathrm{a}-\mathrm{c}$ in patients aged less than 20 years. According to the new criteria, 842 patients could be classified as MFS (83\%), $86 \%$ in the adult cohort and $78 \%$ in the children cohort (Figs 1 and 2). Fiftytwo probands could be classified as ELS (5\%), 31 as MASS (3\%), 5 as MVPS (1\%) and 79 as 
(a)

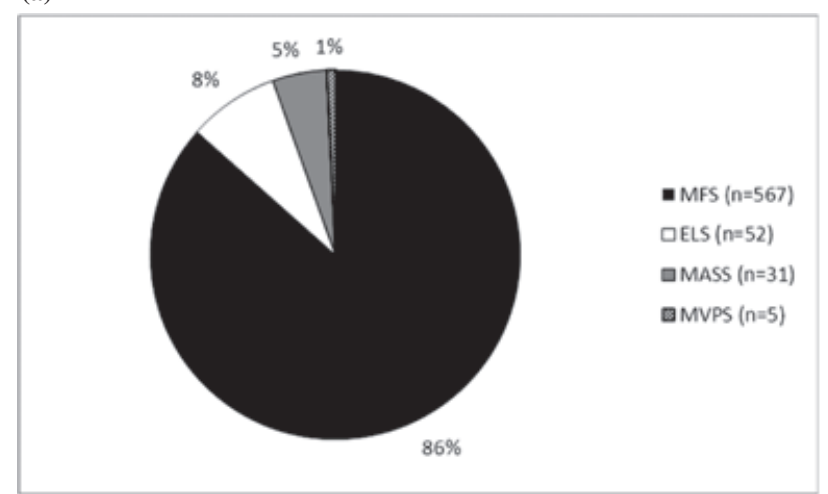

(b)

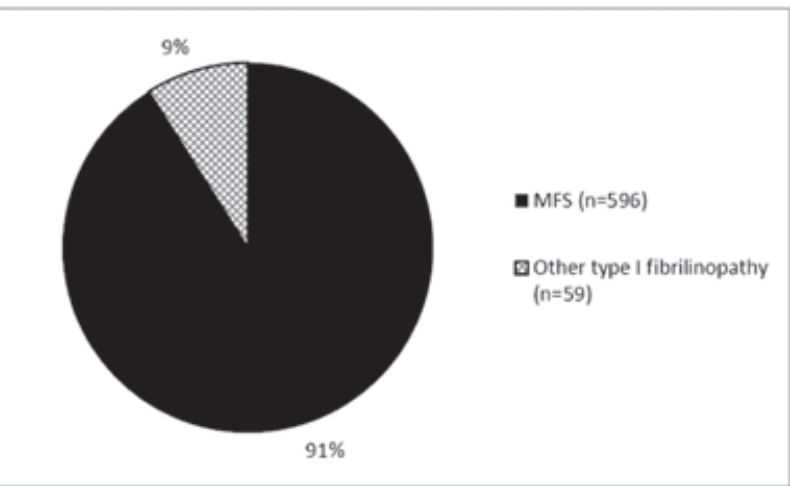

(c)

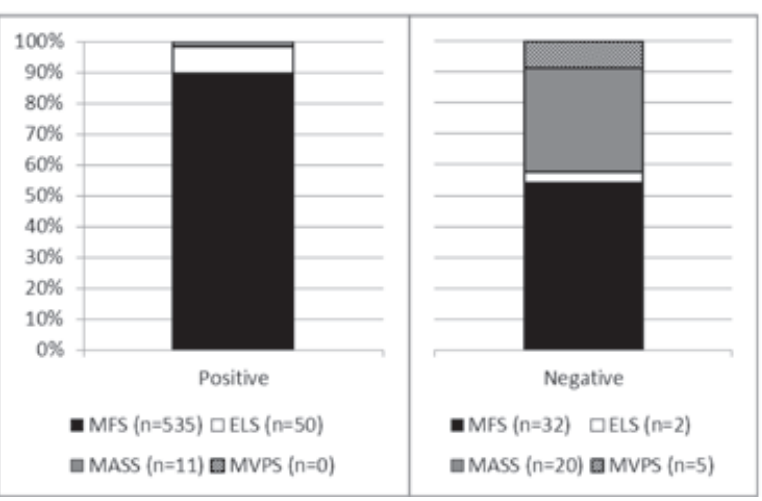

Fig. 1. Diagnosis of MFS or other type I fibrillinopathies in the adult cohort $(n=655)$ according to new Ghent criteria (a) and the old Ghent criteria (b). According to the new Ghent criteria 567 patients were diagnosed with MFS (86\%), 52 patients with ELS (8\%), 31 with MASS (5\%), 5 with MVPS (1\%). According to the old Ghent criteria 596 patients were diagnosed with MFS (91\%) and 59 (9\%) with other type I fibrillinopathies. Reassignment of diagnosis according to the new nosology in adult patients with positive or negative 1996 Ghent criteria (c). In the column of adult patients with positive 1996 Ghent criteria $(n=596)$, 535 keep the same classification, $50(8 \%)$ were reassigned to ELS and $11(2 \%)$ were reassigned to MASS. In the column of patients with negative 1996 Ghent criteria $(n=59), 32(54 \%)$ were reassigned to the diagnosis of MFS, $2(3 \%)$ raise the diagnosis of ELS, $20(34 \%)$ raise the diagnosis of MASS, and $5(8 \%)$ raise the diagnosis of MVPS.

potential MFS (8\%). The median age at diagnosis in patients classified as MFS according to the new nosology was 30 years [IQR 15-41], 36 years [IQR 28.5-44.5] in patients classified as ELS, 29 years [IQR 24-36.5] in patients classified as MASS, 32 years [IQR 26-35] in patients classified as MVPS, and 11 years [IQR 7-16] in patients diagnosed with potential MFS. The number of patients diagnosed with MFS according to the new nosology was slightly lower (842 as compared to 894 according to the 1996 Ghent criteria). However, the distribution differed in $15 \%$ of patients aged 20 years or above (93/655) (Fig. 1). Sixty-one adults, diagnosed with MFS according to the old nosology could now be classified as $\operatorname{ELS}(n=50)$ or MASS $(n=11)$ in the absence of aortic dilatation in the proband or the family. Thirty-two patients who could not be classified as MFS according to the old Ghent criteria were now classified as MFS. This number included 19 patients with aortic dilatation and a systemic score $\leq 5$, without ectopia lentis; 11 patients with ectopia lentis, a mutation that had already been reported in association with aortic dilatation in the UMD-FBN1 database or in another affected family member and a systemic score $\leq 4$, without aortic dilatation; and 2 patients with a systemic score $\geq 7$ without aortic dilatation or ectopia lentis.

The distribution of the systemic score in the cohort is represented in Fig. $3 \mathrm{a}$ in probands aged 20 years or above and in Fig. $3 \mathrm{~b}$ in probands aged less than 20 years. The median systemic score was 7 in both adults [IQR 5-10] and children [IQR 5-8]. The median systemic score was 7 [IQR 5-10] in patients with MFS, 6 [IQR 4.5-8.5] in patients with ELS, 7 [IQR 6.5-9] in patients with MASS, 3 [IQR 3-4] in patients with MVPS, and 7 [IQR 5-8] in patients with potential MFS. Interestingly, 48 patients were classified as MFS although they had a systemic score of less than 3 . Within these patients, 13 had aortic dilatation and ectopia lentis; 25 had aortic dilatation and a family history of MFS; and 10 had ectopia lentis and a family history of MFS and/or FBN1 
(a)

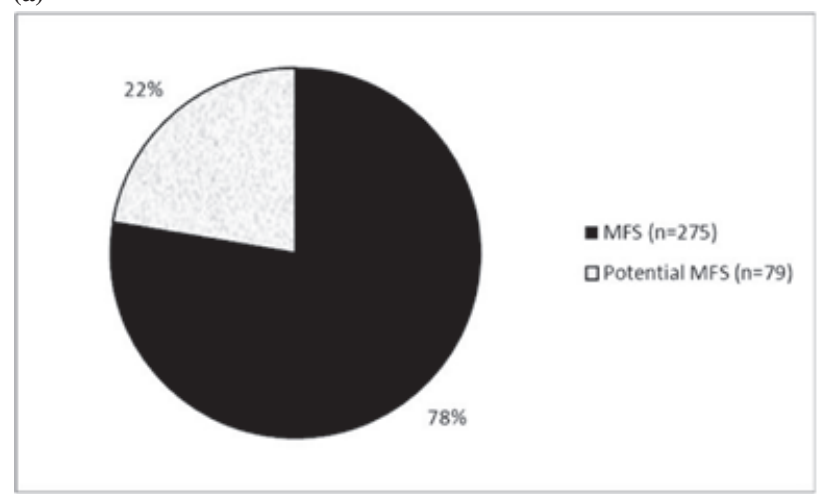

(b)

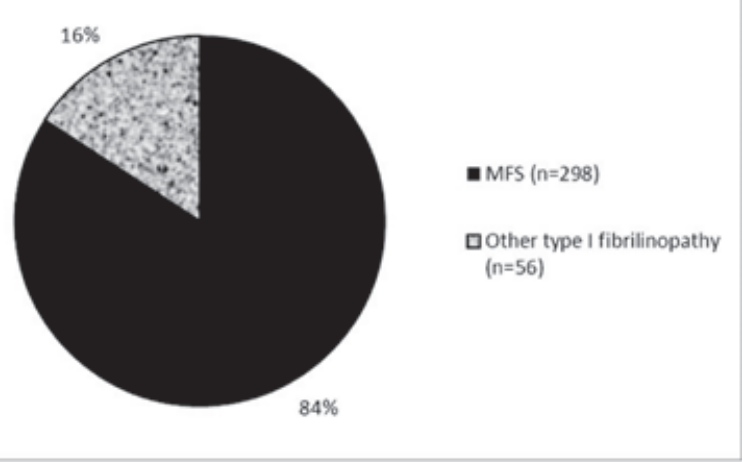

(c)

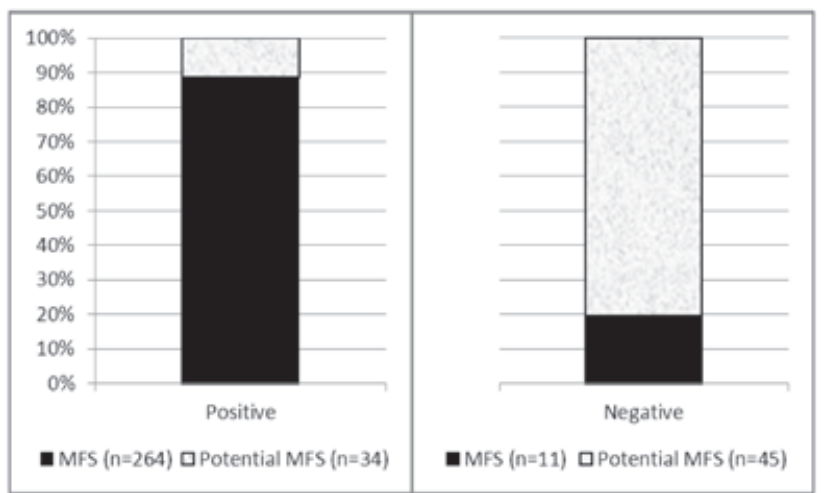

Fig. 2. Diagnosis of MFS or other type I fibrillinopathies in the children cohort $(n=354)$, according to new Ghent criteria (a) and the old Ghent criteria (b). According to the new Ghent criteria 264 patients were diagnosed with MFS (78\%), 79 with potential MFS (22\%). According to the old Ghent criteria 298 patients were diagnosed with MFS (84\%) and 34 (11\%) with other type I fibrillinopathies. Reassignment of diagnosis according to the new nosology in children with positive or negative 1996 Ghent criteria (c). In the column of patients with positive 1996 Ghent criteria $(n=298), 264$ keep the same classification and $34(11 \%)$ were reassigned to potential MFS. In the colomn of patients with negative 1996 Ghent criteria $(n=56), 11(20 \%)$ were reassigned to the diagnosis of MFS and $45(80 \%)$ raise the diagnosis of potential MFS.

mutation already described in association with MFS. However, the role of the systemic score is minor when the presence of the FBN1 mutation is known. Indeed, when considering the items that permitted to reach the diagnosis of MFS according to the new nosology, 409/842 MFS patients had aortic dilatation and ectopia lentis; 394/842 MFS patients had aortic dilatation and family history of MFS; 774/842 MFS patients had aortic dilatation and FBN1 mutation; 479/842 MFS patients had aortic dilatation and a systemic score $\geq 7 ; 228 / 842$ MFS patients had ectopia lentis and a family history of MFS; and 260/842 MFS patients had a systemic score $\geq 7$ and family history of MFS. However, when performing a flow chart, we were able to show that the determination of the systemic score is useful only in $18 / 842(2 \%)$ patients with the diagnosis of MFS.

The distribution of the types of mutations according to the different types of presentation (MFS, ELS, MASS, MVPS and potential MFS) is presented in Table 2. Non-significant differences were found between the different groups of presentation.

\section{Discussion}

In this article, we took advantage of a well-defined series of patients with FBN1 mutation to evaluate the changes encountered by the application of the new 2010 Ghent criteria in the clinical practice (14). The total number of patients diagnosed with MFS according to the 1996 Ghent criteria and the 2010 Ghent criteria slightly differs, but a differential diagnosis could be raised in $15 \%$ of patients. Indeed, the total number of patients diagnosed with MFS according to the new nosology was 842 compared with 894 according to the old 1996 Ghent criteria. The differences are explained by patients who have been reclassified as MFS in the presence of aortic dilatation or EL in a proband with no or minor systemic features when aortic dilatation in an affected relative or in another patient with the same mutation. Also, some patients diagnosed with MFS in the past can now be classified 
(a)

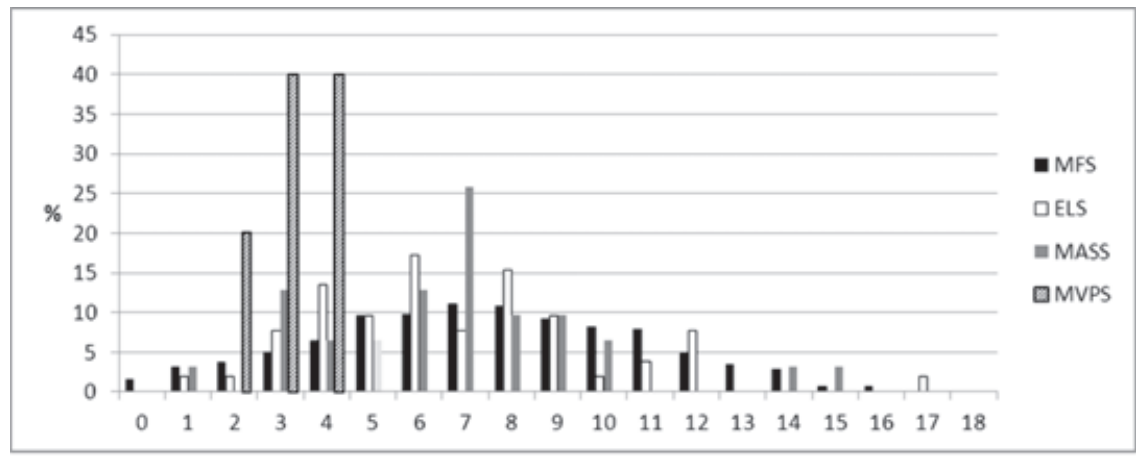

(b)

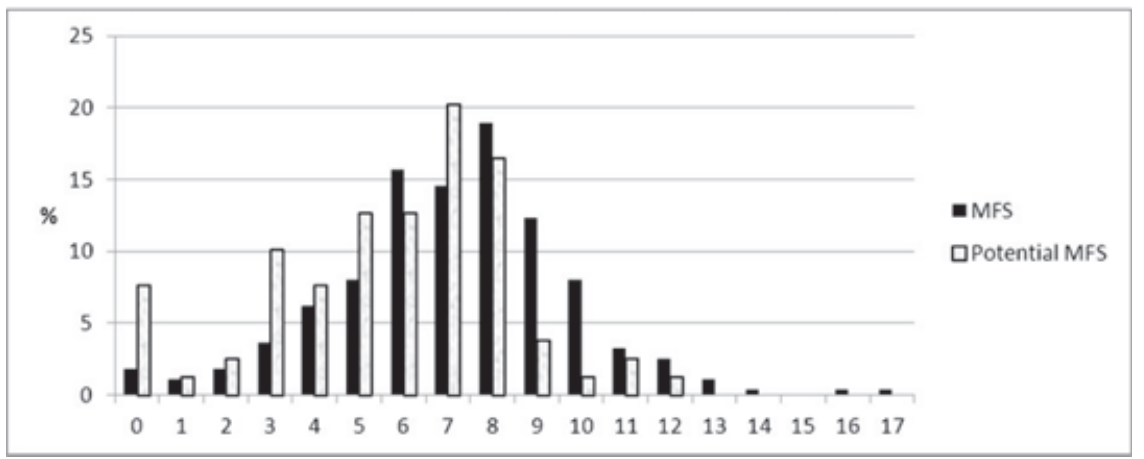

Fig. 3. Distribution of the systemic score in the study cohort $(n=1009)$ in patients with MFS, ELS, MASS and MVPS. (a) Distribution of the systemic score in adults $(\geq 20$ years) $(n=655)$. The median systemic score was 7 [interquartile range (IQR) 5-10] in patients with MFS, 6 [IQR 4.5-8.5] in patients with ELS, 7 [IQR 6.5-9] in patients with MASS, and 3 [IQR 3-4] in patients with MVPS. (b) Distribution of the systemic score in children $(<20$ years $)(n=354)$, in patients with MFS $[n=275$; median age at last follow-up: $10(6-15)]$ and potential MFS $[n=79$; median age at last follow-up: 11 (7-16)]. The median systemic score was 7 [IQR 5-8] in both patients with MFS and potential MFS.

Table 2. Repartition of mutation in the different types of presentation

\begin{tabular}{lccccc}
\hline & $\begin{array}{c}\text { Nonsense } \\
(n=137)\end{array}$ & $\begin{array}{c}\text { Frameshift } \\
(n=170)\end{array}$ & $\begin{array}{c}\text { Splicing } \\
(n=110)\end{array}$ & $\begin{array}{c}\text { Missense } \\
(n=475)\end{array}$ & $\begin{array}{c}\text { Inframe deletion/ } \\
\text { insertion }(n=20)\end{array}$ \\
\hline MFS (\%) & 15 & 17 & 10 & 56 & 2 \\
ELS (\%) & 6 & 17 & 10 & 63 & 4 \\
MASS (\%) & 16 & 19 & 0 & 55 & 3 \\
MVPS (\%) & 0 & 0 & 20 & 100 & 0 \\
Potential MFS (\%) & 8 & 15 & 53 & 4 \\
\hline
\end{tabular}

ELS, ectopia lentis syndrome; MASS, mitral valve, aorta, skeleton and skin; MFS, Marfan syndrome; MVPS, mitral valve prolapse syndrome.

as ELS or MASS in the absence of aortic dilatation in the proband, an affected family member, or in other patients with the same mutation. As with the 1996 Ghent criteria, special consideration should be given to children ( $<20$ years) because some clinical features may appear later in life, and this population has been analyzed separately in this study.

We found that the new nosology offers some advantages: (i) the nosology may be easier to apply in a number of cases because the presence of aortic dilatation and EL or aortic dilatation and FBN1 mutation is sufficient to diagnose MFS (Fig. 4), and when the calculation of the systemic score is needed, several of the 'minor' criteria often difficult to assess have been eliminated; (ii) the number of possible diagnoses may be more representative of the clinical extent or severity of the condition; (iii) the systemic score can be interpreted as a severity score for non-aortic/eye manifestations, which can be an advantage for future studies (genotype/phenotype, variable expressivity, modifier genes); and (iv) the identification of ELS now makes sense because the new genes recently associated with EL, with or without some skeletal features of the MFS spectrum, are taken into 


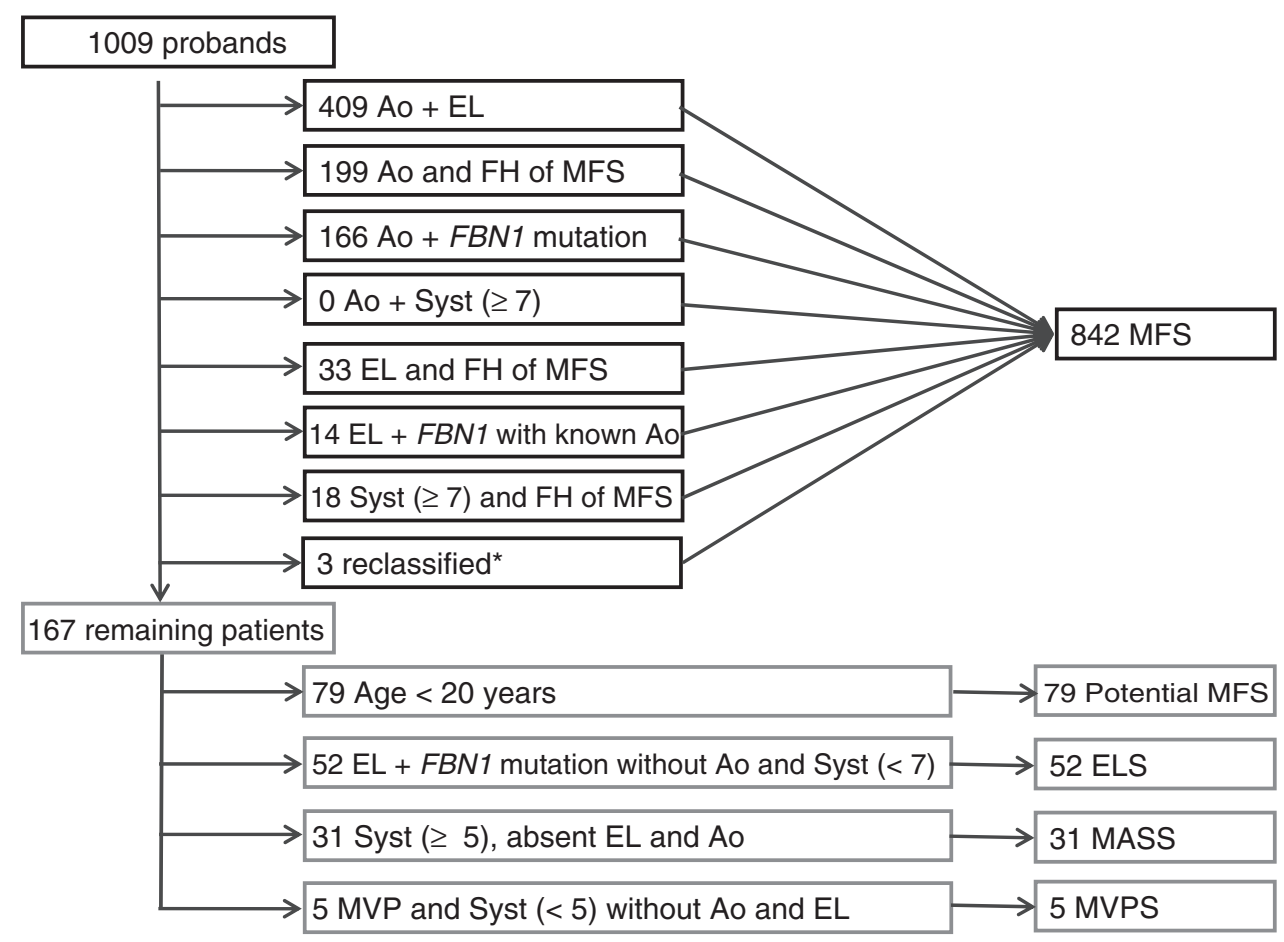

Fig. 4. Flow chart of the application of the new criteria in the present series. Ao, aortic dilatation/dissection; EL, ectopia lentis; FH, family history; Syst, systemic score; FBN1, fibrillin-1 mutation; FBN1 with known Ao, FBN1 mutation that has been identified in an individual with aortic aneurysm; MASS, myopia, mitral valve prolapse, aortic root dilation, skeletal findings, striae syndrome; ELS, ectopia lentis syndrome; MFS, Marfan syndrome. ${ }^{*}$ Correspond to patients who have been reclassified to MFS in the presence of a systemic score $<7$ but familial history of MFS with FBN1 mutation.

account $(27,28)$. Of notice, not all clinicians have mutation analysis available, because of the variable availability of molecular testing across countries and delay for the results.

However, it raises some comments: (i) When a patient is diagnosed with another condition in the absence of aortic dilatation (ELS, MASS, MVPS), physicians need to be aware that regular follow-up including annual cardiovascular imaging is advised to monitor aortic size over time, because it has been shown that aortic dilatation can appear at all ages (23-29). Reclassification to MFS may occur if aortic dilatation appears during follow-up, as shown in the past $(30,31)$. Counseling for patients with either ELS or MASS phenotype should include the risk of a more severe presentation in their offspring, including aortic enlargement. (ii) Besides easier applicability in general, when a mutation is found in a patient with EL or a systemic score $\geq 7$ but without aortic dilatation, the clinician needs to have access to a database to know whether the mutation has already been reported in association with aortic dilatation, thus raising the possible diagnosis of MFS, reinforcing the importance of up-todate UMD database (19). The new nosology also introduces the $Z$-score calculation in the new nosology $v s$ 'aortic dilatation' in the old nosology. There are different ways of measuring $Z$-scores for aortic dilatation world-wide; therefore, all patients within a group may not necessarily be truly comparable from the point of view of their aortic phenotype. (iii) If the new nosology is strictly applied, there is a gap for patients with FBN1 mutation, a family history of MFS but a systemic score $<7$, and for patients with a systemic score $\geq 7$, an FBN1 mutation known to cause aorta pathology without family history. We propose that an adaptation of the criteria should be made so that such patients should raise the diagnosis of MFS.

This study presents two sources of bias. The first is due to the inclusion criteria of the studied cohort, namely the presence of FBN1 mutation. However, as FBN1 mutations have been associated with an aortic risk (12), it is important to evaluate how often MFS criteria effectively diagnose the aortic risk in this population. The second source of bias is due to the retrospective nature of the study, which made it impossible to score certain items precisely. The authors' choice in analyzing the systemic scores with doubtful items is quite arbitrary. However, we showed that the utility of the systemic score was minor in the present series. 
Similar remarks can be made regarding the $Z$ score for the determination of aortic dilatation. Indeed, Radonic et al. (32) recently reported in 2011 that the diagnosis of MFS according to the 1996 nosology was rejected in 13/180 patients because the $Z$-score of the aortic root was $<2$, although the aortic diameter was larger than 40 $\mathrm{mm}$ in six of them. Another point was also raised from Radonic study. The authors also showed that the new nosology permitted to revise the diagnosis in patients with an aorta dilatation and dural ectasia but no FBN1 mutation who have been diagnosed incorrectly as MFS in the past as too much weight has been given to dural ectasia.

In conclusion, if the new Ghent criteria are applied with common sense and flexibility, they should help and support clinicians who are less experienced with the Marfan phenotype. Indeed, even if the diagnosis of MFS is not reached, regular aortic follow-up is mandatory in every patient with a diagnosis of ELS, MASS or MVPS. A longitudinal study for the follow-up of patients with such alternative diagnoses is required to determine the proportion of patients who will meet the criteria for MFS later in life.

\section{Acknowledgements}

This work was supported by a grant from the Association Française pour les Syndromes de Marfan et Apparentés (AFSMA 2010), the General Counsil of Burgundy, French ministry of health (PHRC 2004, 2009), GIS maladies rares 2004, Bourse de la Société Francaise de Cardiologie, Fédération Française de Cardiologie 2005, and ANR-05-PCOD-014, GDPM-2.

\section{References}

1. Pyeritz RE. Marfan syndrome: current and future clinical and genetic management of cardiovascular manifestations. Semin Thorac Cardiovasc Surg 1993: 5: 11-16.

2. Arslan-Kirchner M, Arbustini E, Boileau C et al. Clinical utility gene card for: Marfan syndrome type 1 and related phenotypes [FBN1]. Eur J Hum Genet 2011. DOI: 10.1038/ejhg.2011.68.

3. Judge DP, Dietz HC. Marfan's syndrome. Lancet 2005: 366: $1965-1976$

4. Beighton P, de Paepe A, Danks D et al. International Nosology of Heritable Disorders of Connective Tissue, Berlin, 1986. Am J Med Genet 1988: 29: 581-594.

5. De Paepe A, Devereux RB, Dietz HC, Hennekam RC, Pyeritz RE. Revised diagnostic criteria for the Marfan syndrome. Am J Med Genet 1996: 62: 417-426.

6. Pereira L, Levran O, Ramirez F et al. A molecular approach to the stratification of cardiovascular risk in families with Marfan's syndrome. N Engl J Med 1994: 331: 148-153.

7. Nijbroek G, Sood S, McIntosh I et al. Fifteen novel FBN1 mutations causing Marfan syndrome detected by heteroduplex analysis of genomic amplicons. Am J Hum Genet 1995: 57: $8-21$.

8. Loeys B, Nuytinck L, Delvaux I, De Bie S, De Paepe A. Genotype and phenotype analysis of 171 patients referred for molecular study of the fibrillin-1 gene FBN1 because of suspected Marfan syndrome. Arch Intern Med 2001: 161: $2447-2454$

9. Loeys B, De Backer J, Van Acker P et al. Comprehensive molecular screening of the FBN1 gene favors locus homogeneity of classical Marfan syndrome. Hum Mutat 2004: 24: 140-146.

10. Stheneur C, Collod-Béroud G, Faivre L et al. Identification of the minimal combination of clinical features in probands for efficient mutation detection in the FBN1 gene. Eur J Hum Genet 2009: 17: 1121-1128.

11. Faivre L, Masurel-Paulet A, Collod-Béroud G et al. Clinical and molecular study of 320 children with Marfan syndrome and related type I fibrillinopathies in a series of 1009 probands with pathogenic FBN1 mutations. Pediatrics 2009: 123: 391-398.

12. Faivre L, Collod-Beroud G, Child A et al. Contribution of molecular analyses in diagnosing Marfan syndrome and type I fibrillinopathies: an international study of 1009 probands. J Med Genet 2008: 45: 384-390.

13. De Backer J, Loeys B, Devos D, Dietz H, De Sutter J, De Paepe A. A critical analysis of minor cardiovascular criteria in the diagnostic evaluation of patients with Marfan syndrome. Genet Med 2006: 8: 401-408.

14. Loeys BL, Dietz HC, Braverman AC et al. Towards a revised Ghent nosology for the Marfan syndrome. J Med Genet 2010: 47: 476-485.

15. Mizuguchi T, Collod-Beroud G, Akiyama T et al. Heterozygous TGFBR2 mutations in Marfan syndrome. Nat Genet 2004: 36: 855-860.

16. Loeys BL, Chen J, Neptune ER et al. A syndrome of altered cardiovascular, craniofacial, neurocognitive and skeletal development caused by mutations in TGFBR1 or TGFBR2. Nat Genet 2005: 37: 275-281.

17. Faivre L, Collod-Beroud G, Loeys BL et al. Effect of mutation type and location on clinical outcome in 1,013 probands with Marfan syndrome or related phenotypes and FBN1 mutations: an international study. Am J Hum Genet 2007: 81: 454-466.

18. Loeys BL, Schwarze U, Holm T et al. Aneurysm syndromes caused by mutations in the TGF-beta receptor. N Engl J Med 2006: 355: 788-798.

19. Collod-Beroud G, Le Bourdelles S, Adès L et al. Update of the UMD-FBN1 mutation database and creation of an FBN1 polymorphism database. Hum Mutat 2003: 22: 199-208.

20. Frédéric MY, Boileau C, Hamroun D, Claustres M, Béroud C, Collod-Béroud G. UMD-predictor, a new prediction tool for nucleotide substitution pathogenicity; application to four genes: FBN1, FBN2, TGFBR1 and TGFBR2. Hum Mut 2009: 30: $952-959$.

21. Faivre L, Collod-Beroud G, Callewaert B et al. Pathogenic FBN1 mutations in 146 adults not meeting clinical diagnostic criteria for Marfan syndrome: further delineation of type 1 fibrillinopathies and focus on patients with an isolated major criterion. Am J Med Genet A 2009: 149A: 854-860.

22. Faivre L, Collod-Beroud G, Callewaert B et al. Clinical and mutation-type analysis from an international series of 198 probands with a pathogenic FBN1 exons 24-32 mutation. Eur J Hum Genet 2009: 17: 491-501.

23. Détaint Faivre D, Collod-Beroud L, Child G et al. G Cardiovascular manifestations in men and women carrying a FBN1 mutation. Eur Heart J 2010: 31: 2223-2229.

24. Faivre L, Dollfus H, Lyonnet $\mathrm{S}$ et al. Clinical homogeneity and genetic heterogeneity in Weill-Marchesani syndrome. Am J Med Genet A 2003: 123A: 204-207. 
25. Faivre L, Gorlin RJ, Wirtz MK et al. In frame fibrillin-1 gene deletion in autosomal dominant Weill-Marchesani syndrome. J Med Genet 2003: 40: 34-36.

26. Dagoneau N, Benoist-Lasselin C, Huber C et al. ADAMTS10 mutations in autosomal recessive Weill-Marchesani syndrome. Am J Hum Genet 2004: 75: 801-806.

27. Ahram D, Sato TS, Kohilan A et al. A homozygous mutation in ADAMTSL4 causes autosomal-recessive isolated ectopia lentis. Am J Hum Genet 2009: 84: 274-278.

28. Morales J, Al-Sharif L, Khalil DS et al. Homozygous mutations in ADAMTS10 and ADAMTS17 cause lenticular myopia, ectopia lentis, glaucoma, spherophakia, and short stature. Am J Hum Genet 2009: 85: 558568 .
29. Rybczynski M, Bernhardt AM, Rehder U et al. The spectrum of syndromes and manifestations in individuals screened for suspected Marfan syndrome. Am J Med Genet A 2008: 146A: 3157-3166.

30. Kainulainen K, Karttunen L, Puhakka L, Sakai L, Peltonen L. Mutations in the fibrillin gene responsible for dominant ectopia lentis and neonatal Marfan syndrome. Nat Genet 1994: 6: 64-69.

31. Black C, Withers A, Gray JR et al. Correlation of a recurrent FBN1 mutation (R122C) with an atypical familial Marfan syndrome phenotype. Hum Mut 1998: (Suppl 1): S198-S200.

32. Radonic T, de Witte $\mathrm{P}$, Groenink $\mathrm{M}$ et al. Critical appraisal of the revised Ghent criteria for diagnosis of Marfan syndrome. Clin Genet 2011. DOI: 10.1111/j.1399-0004.2011.01646.x. 